\title{
IMPLEMENTATION OF MTI RADARS USING NEURAL NETWORK
}

\author{
Fawzy Ibrahim *
}

\begin{abstract}
The purpose of moving target indicator (MTI) radars is to reject signals from fixed unwanted targets, such as buildings, hills, and trees, and retain signals for detection of moving targets such as aircraft, ships, missiles,..etc. There are many conventional MTI techniques such as, the adaptive pulse canceller, doppler filter banks, and fast Fourier transform (FFT) filter banks. Each technique suffers certain considerable limitation. This paper utilizes a neural network approach to implement a system that can be used to detect moving targets in severely cluttered environment. A three-layer neural network has been trained to perform as moving target indicator. The backpropagation training algorithm has been used to train the proposed network. Significant performance improvements have been achieved as compared with the conventional fast Fourier transform method.
\end{abstract}

\section{INTRODUCTION}

The basic function of pulse radars is to detect a target by transmitting a pulse of radio frequency energy and receiving a portion of the reflected energy from the target. Simple low pulse repetition frequency (PRF) radars measure the range or the distance between the radar and the target by transmitting a pulse and timing the returned pulse from the target as shown in Fig. 1. In airborne applications, pulse radar encounters difficulty in detecting targets under "look down" conditions as shown in Fig. 2 (b). It is seen that the ground returns emanate from ranges equal to aircraft altitude to ranges exceeding the slant range to the ground along the main beam. Most, of these returns are due to antenna sidelobes. This makes the detection process under "look down" conditions difficult; therefore, the desire for reliable look-down detection capability together with the requirements of increased target detection range led to the design of doppler radars $[1-3]$.

* Lecturer, Department of Electrical and Computer Engineering Military Technical College, Kobry El-Koba, Cairo, Egypt. 
Using the doppler frequency effect, rather than timing of the transmit-receive, allows one to use a very high PRF which gives the range rate as a function of transmit-receive frequency shift, as shown in Fig. 2 (c). The increased number of pulses in high PRF radars compromises the ability of the radar to determine radar-target range by simple time discrimination ranging [1-3]]. For this reason medium PRF radar system can be designed to have a greater degree of tactical flexibility in target detection by combining the desirable feature of both low and high PRF radars. The medium PRF radar systems use time discrimination for ranging and frequency discrimination for range rate calculations. The clutter foldover effect caused by ambiguous range makes the medium PRF radar requires greater clutter rejection capability.

In this paper, the neural network concept is applied for moving target detection of the medium PRF radar in sever clutter environment. the performance of the neural network is compared with that of the conventional filter bank method. The neural network approach shows better performance especially in highly cluttered environment.

\section{TRADITIONAL MTI TECHNIQUES}

The phenomenon used in MTI radar is that targets moving with finite radial velocities produce doppler-shifted echoes. The doppler information can be used to discriminate in favor of moving targets such as aircraft, and against fixed targets and clutter. The target doppler frequency $\left(f_{d}\right)$ can be expressed as:

$$
\mathrm{f}_{\mathrm{d}}=\left(2 \mathrm{v}_{\mathrm{r}} / \lambda\right) \cos \zeta
$$

where $v_{i}$ is the target relative velocity, $\zeta$, is the angle between the target velocity vector and radar-target line of $\mathrm{sight}$, and $\lambda$ is the radar wavelength. Fig. 3 shows a typical medium PRF radar frequency spectrum in the presence of ground clutter. Therefore, the major function of a MTI radar is to detect the target doppler shift from sever clutter environment.

Adaptive pulse cancellers process the received signals in the time domain. Based on the clutter information the adaptive pulse canceller can only distinguish whether this is a moving target or clutter. The target velocity can not be obtained from pulse canceller directly. In many application situations the target velocity is required. Therefore in advanced radar systems, filter banks are used to further process the output signals from pulse cancellers. 
Another technique is to use doppler filter banks directly without using pulse cancellers. Doppler filter bank technique is used in many modern radar systems to detect not only moving targets but also their associated radial velocities. Discrete Fourier transform (DFT) and the fast Fourier transform (FFT) are the most commonly used technique to construct filter banks for target velocity detection and clutter rejection. Based on the sampling theory, the maximum detectable target velocity is that corresponding to a doppler frequency of $1 / 2 *$ PRF. A filter bank covering frequency from $-1 / 2 *$ PRF to $1 / 2 *$ PRF can be constructed from a direct application of fast Fourier transformation. There are many other ways to form filter banks, such as using a set of narrow bandpass filters in parallel. But the most economical and reliable one is to use FFT.

\section{MOTIVATION}

Each of the conventional MTI techniques discussed in the previous section suffers some limitations. These limitations may include [5]:

1. The computation load is so huge when applying the DFT.

2. The number of stages used in the FFT is $\log _{2} n$, where $n$ is the number of points and has to be integer and power of 2 .

3. The spectral leakage effect due to the finite window length in observation will degrade the signal strength in the mainlobe and enhance the signal strength in the sidelobes.

4. The signal magnitude in the sidelobes can be reduced by using many windowing schemes such as Hanning, Hamming, Blackman, and Barlett window [5]. However, all these windows can greatly reduce the signal magnitude in the sidelobes, but the mainlobe bandwidth has been increased. In other words, the suppression of the sidelobe signals scarifies the resolution due to the widening of mainlobe bandwidth.

These limitations were the motivation to initiate this work.

\section{MTI NEURAL NETWORK STRUCTURE AND TRAINING}

Fig. 4 shows the structure of the proposed filter, which is a three-layer feedforawd neural network. The system network has 16 neurons in the input layer, 24 neurons in the hidden layer, and 15 neurons in the output layer. The connection between the input and the hidden layer is a $16 * 24$ array of weights $W$ and the connection between the hidden and the output layer is a $24 * 15$ array of weights $K$. The backpropagation training algorithm [6-8] has been used to train the proposed network. 
The objective of training the network is to adjust the weight matrices $W$ and $K$ so that application of a set of inputs produces the desired set of outputs. Training assumes that every input vector is paired with a target vector representing the desired output, together these are called a training pair. Usually a network is trained over a number of training pairs.

In our case, for each input signal (pattern), two corresponding output neurons will be activated to generate output signals with magnitude close to 1 , and the rest of neurons in the output layer generate almost zero output. The output neurons are numbered, as shown in Fig. 4, from $-7,-6, \ldots, 0, \ldots, 6,7$, then training pattern 1 will activate output neurons -1 and 1 , training pattern 2 will activate output neurons -2 and 2 , and so forth. Training pattern 8 is used to activate output neuron 0 only.

Before starting the training process, all of weights must be initialized to small random numbers. This ensures that the network is not saturated by large values of the weights. Training the backpropagation network requires the steps that follow [9]:

1. Select a training pair from a training set.

2. Apply the input vector to network input.

3. Calculate the output of the network.

4. Calculate the error between the network output and the desired output.

5. Adjust the weights of the network in a way that minimizes the error.

6. Select the next training pair and repeat steps 2 through 5 for each vector in the training set until the error for the entire set is acceptably low.

Physically this three layer neural network shown in Fig. 4 is trained to perform as a set of bandpass filters with different center frequencies. The output of neuron $n$ and $-n$ are corresponding to a bandpass filter with center frequency at ( $n / 16$ ) times the sampling frequency, where $n$ varies from 0 to 7 . For a pulse doppler radar system, the sampling frequency, $F$, is its pulse PRF.

\section{SIMULATION RESULTS}

The simulation work for training the proposed neural network was conducted using the NETS package [10], while the stochastic modeling was done using the signal processing package [11]. Both packages are runable on the IBM personal computer. 
The performance of the neural network is tested with randomly sampled input signals to present different moving targets with different velocities. The white Gaussian noise has been used to simulate the highly cluttered environments. For the purpose of comparison, the output from the FFT filter bank method have been calculated. Fig. 5 shows the output comparison between the neural network and the FFT for different doppler signals. From this figure, It is clear that the neural network performs much better in clutter suppression than the conventional FFT filter bank method. In addition, it is also noted that the neural network mainlobe width remains unchanged, implying that the target velocity detection resolution will not be changed after the suppression of the signal magnitude in the sidelobes. this feature can not be achieved through the windowed FFT method (such as Blackman, hamming,...,etc) that can only suppress the signal magnitude in the side lobes at the expense of widening the mainlobe [5], which means that the detection resolution is reduced.

It should be noted that the neural network takes a very long time to be trained (usually overnight); However, once trained, it takes a short time to retrieve or respond to a given input (in the order of few msecs).

\section{CONCLUSION}

The rapid development of new massively parallel neural network hardware offers the opportunity for implementing special purpose detection processing which has the potential for considerable performance gains. This work has shown that a three layer feedforward neural network can be trained to detect moving targets in very cluttered environments. The proposed network performs much better than the conventional FFT method in several aspects which may include: suppression of the sidelobe clutter without widening the mainlobe bandwidth, application of any number of the input signal samples, and reduction of the computational complexity. 


\section{REFERENCES}

1. Skolnik,M.E., "Radar handbook, " MaGraw-hill book company, 1990.

2. Hovanessian, S.A., "Radar system design and analysis," Artech House, Inc 1984 .

3. Lynn,P.A.,"Radar systems" Van Nostrand Reinhold, 1987.

4. Swartzlander, E. E.," VLSI Signal Processing Systems, "Kluwer Academic publisher, 1986.

5. Oppenheim, A. V. and Schaferr R.W., " Discrete-Time Signal Processing, "Printice-Hall 1989.

6. Freeman, J.A. and Skapura, D.M.," Neural Networks:

Algorithms, Applications and Programming Techniques," AddisonWesely Publishing company, Inc. 1991.

7. Lippmann, R.P., "An Introduction to Computing with Neural Nets," IEEE ASSP Mag, PP. 4-22, April 1987.

8. Margon, D.P. and Scofiled, "Neural Networks and Speech Processing,"Kluwer Academic Publishers, 1991.

9. McClelland, J.L and Rumelhart, D.E., "Explorations in Parallel Distributed Processing: A Handbook of Models, Programs, and Exercises, "The Massachusetts Institute of Technology, 1988 .

10. Baffes, P., " Nets User's Guide, " Lyndon B. Jonson Space Center. U. S. A., 1989.

11. Stearns, S.D. and David, R.A.," Signal Processing Algorithms, "Printice-Hall. Inc. 1987.

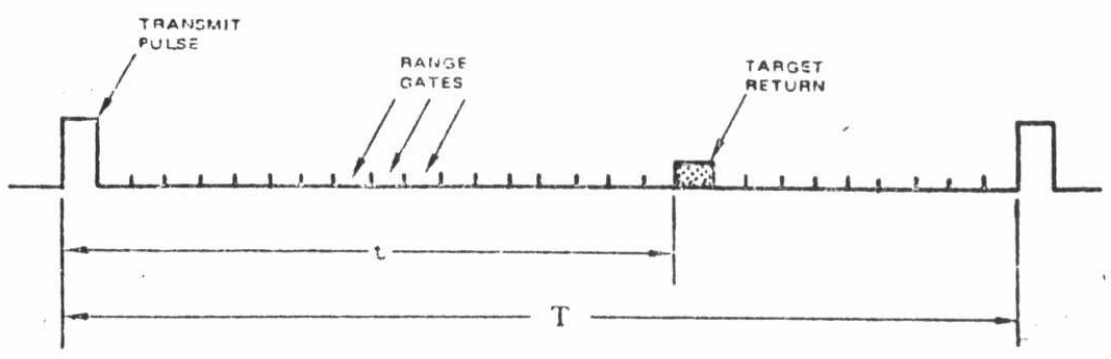

Fig. 1 Pulse radar measurement 


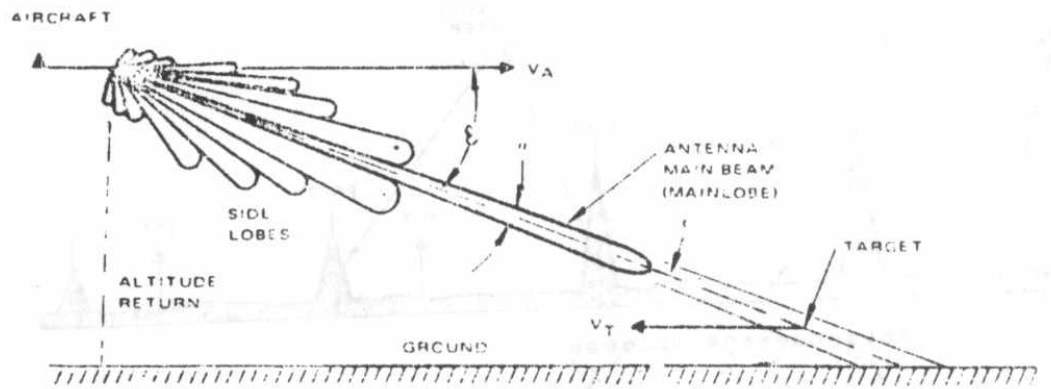

(a) Look down condition.

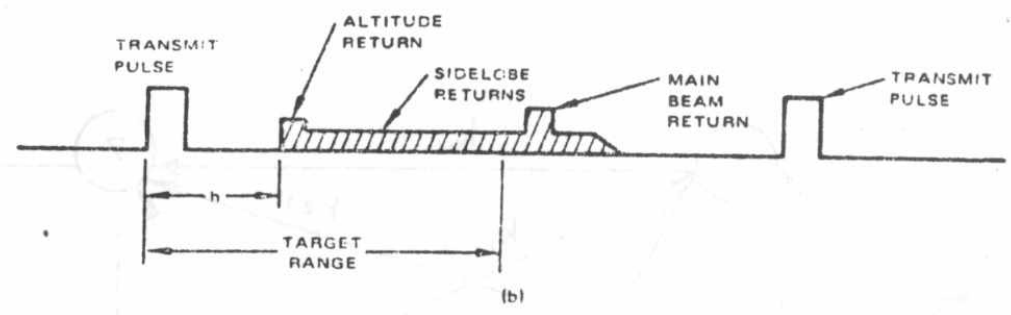

(b) Ground returns.

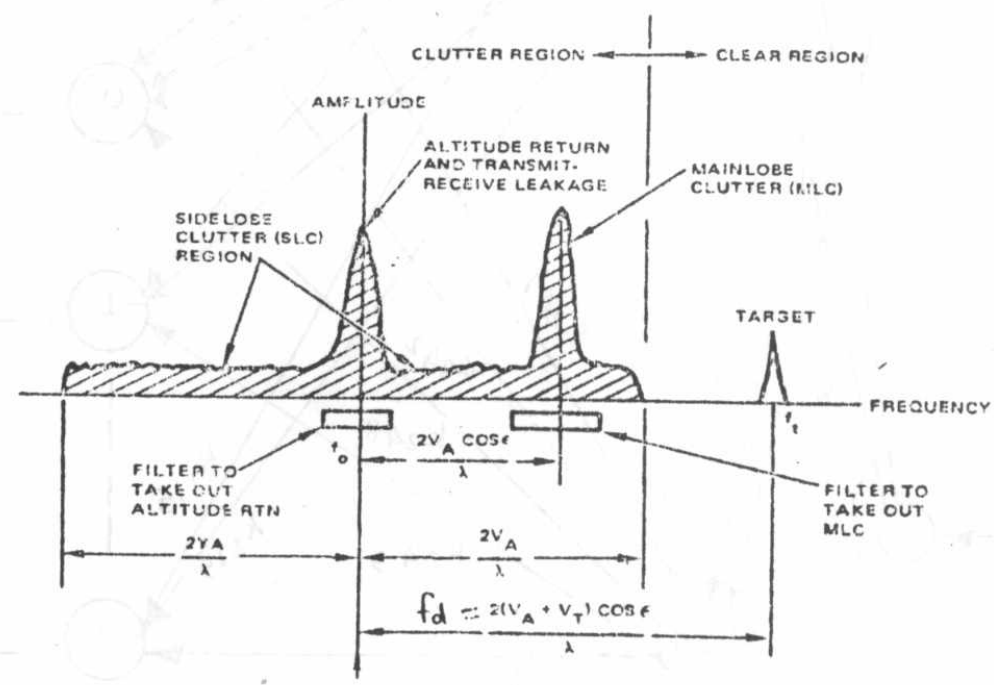

(c) Amplitude-frequency characteristics of a doppler radar.

Fig. 2 Airborne pulse radar detection of ground targets. 


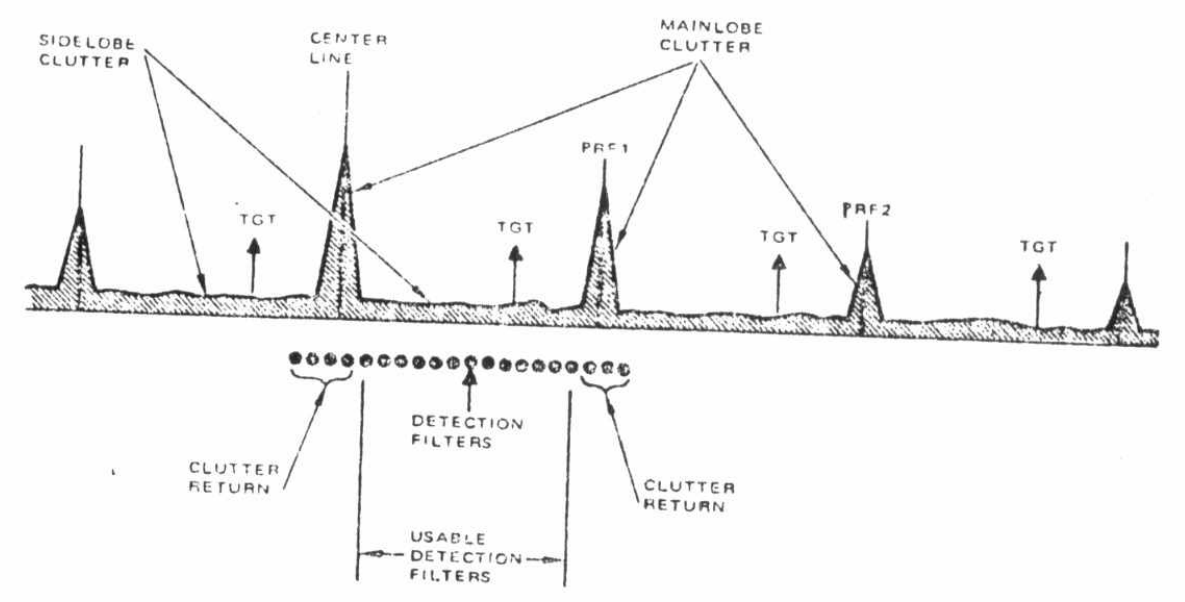

Fig. 3 Medium PRF radar frequency in presence of ground clutter.

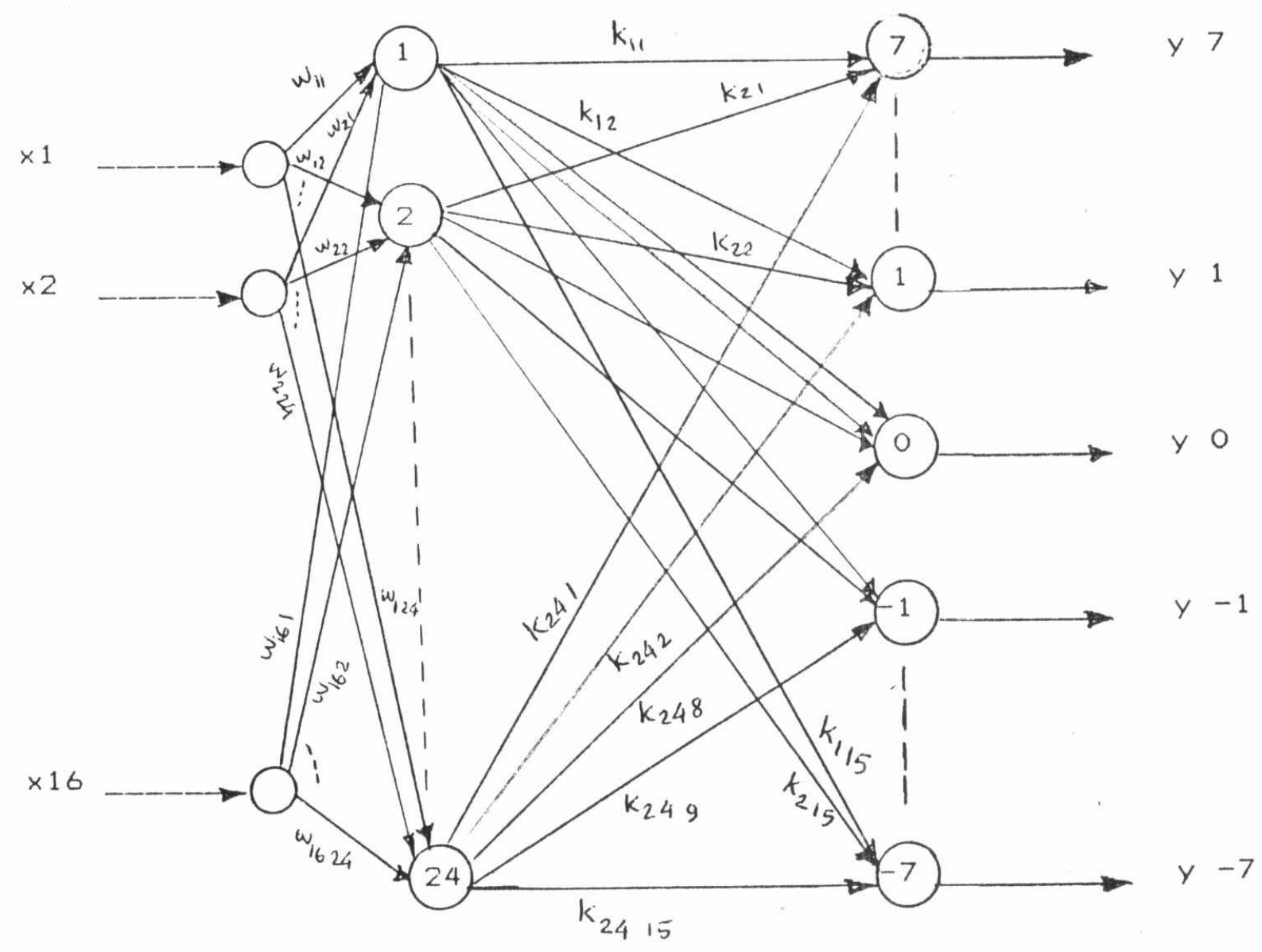

Input layer

First layer
24 neurons

Output layer

15 neurons

Fig. 4 The proposed neural network MTI filter. 

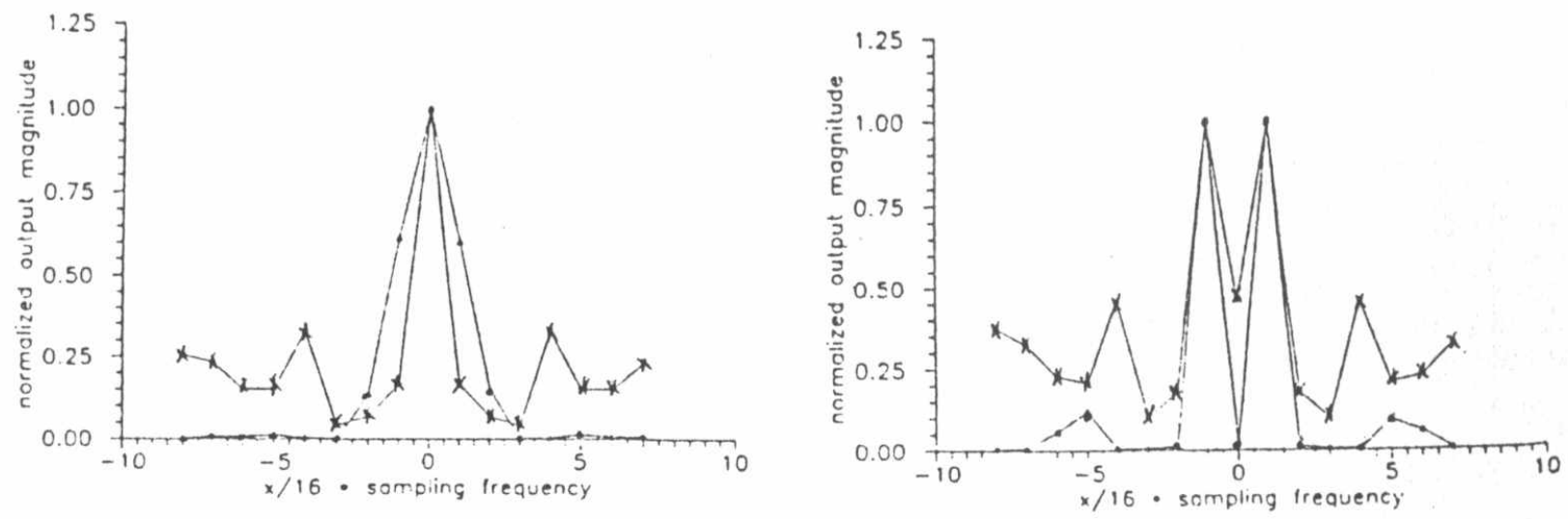

(a) $f_{d}=(0.25 / 16) * F_{0}$

(b) $f_{d}=(1.23 / 16) * F_{*}$
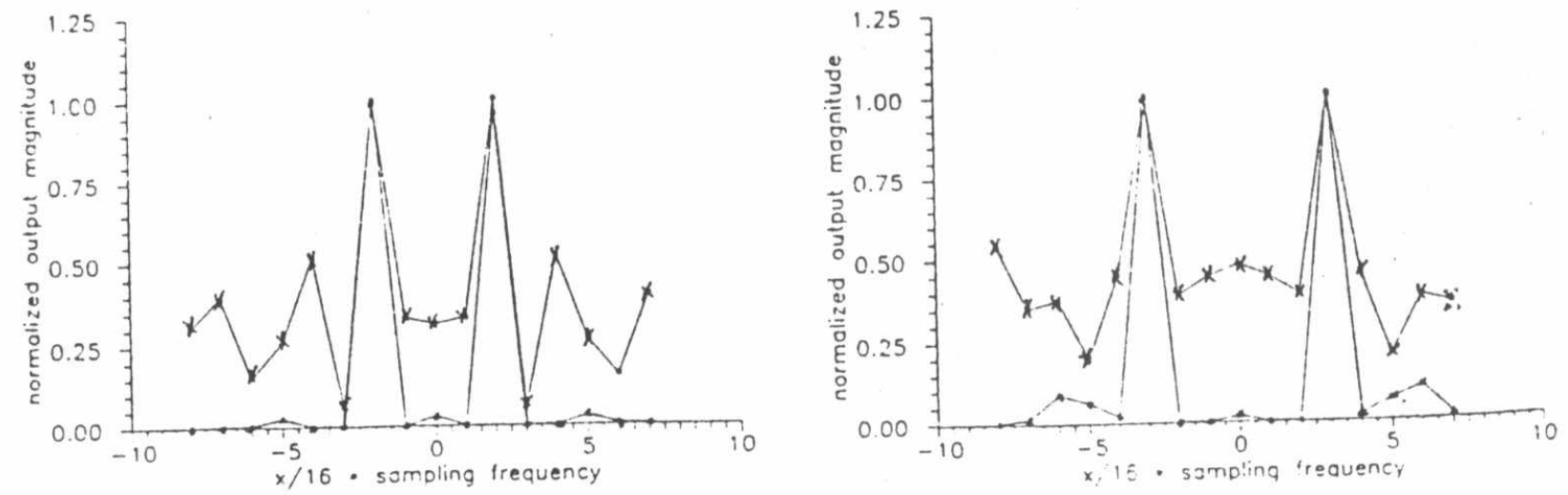

(c) $f_{d}=(2.48 / 16) * F_{0}$

(d) $f_{d}=(3.33 / 16) * F_{*}$
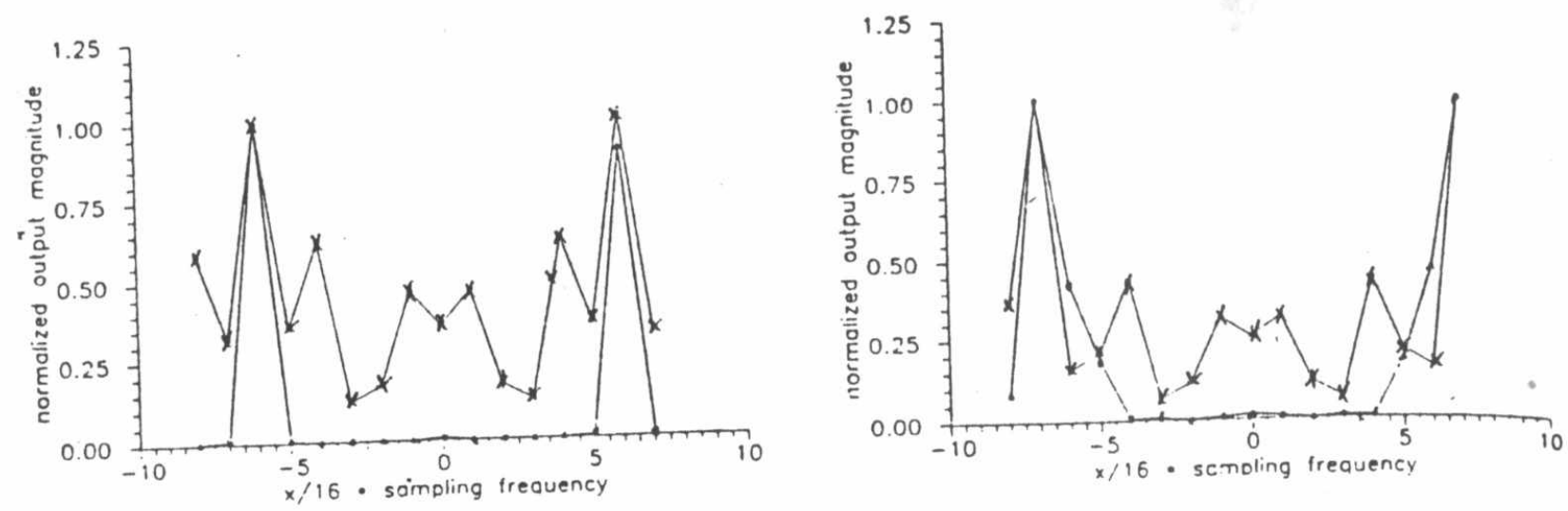

(e) $f_{d}=(6.18 / 16) * F_{*}$

(f) $f_{d}=(7.10 / 16) * F_{*}$

Fig. 5 Performance comparison between neural network and FFT outputs for different frequencies and $s / N=0 \mathrm{~dB}$.

-.... Neural network output.

$-x_{* * *}^{*}$ 16-point FFT output. 